\title{
Evaluation of Amniotic-Derived Membrane Biomaterial as an Adjunct for Repair of Critical Sized Bone Defects
}

\author{
Mikael Starecki, ${ }^{1}$ John A. Schwartz, ${ }^{1}$ and Daniel A. Grande ${ }^{1,2}$ \\ ${ }^{1}$ Department of Orthopaedic Surgery, North Shore/LIJ Health System, 300 Community Drive, Manhasset, NY 11030, USA \\ ${ }^{2}$ Orthopaedic Research Laboratory, Feinstein Institute for Medical Research, 350 Community Drive, Manhasset, NY 11030, USA
}

Correspondence should be addressed to Daniel A. Grande; dgrande@nshs.edu

Received 12 September 2013; Accepted 9 March 2014; Published 2 April 2014

Academic Editor: Vinod K. Panchbhavi

Copyright () 2014 Mikael Starecki et al. This is an open access article distributed under the Creative Commons Attribution License, which permits unrestricted use, distribution, and reproduction in any medium, provided the original work is properly cited.

\begin{abstract}
Introduction. Autogenous bone graft is the gold standard in reconstruction of bone defects. The use of autogenous bone graft is problematic because of limited bone as well as donor site morbidity. This study evaluates a novel biomaterial as an alternative to autogenous bone graft. The biomaterial is amniotic membrane, rich in growth factors. Methods. Twenty-one adult male SpragueDawley rats were implanted with biomaterial using the rat critical size femoral gap model. After creation of the critical size femoral gap animals were randomized to one of the following groups: Group 1 (control): gap left empty and received no treatment; Group 2 (experimental): the gap was filled with commercially available bone graft; Group 3 (experimental): the gap was filled with bone graft plus NuCel amniotic tissue preparation. Results. The experimental groups demonstrated new bone formation compared to controls. The results were evident on radiographs and histology. Histology showed Group 1 controls to have 11.1\% new bone formation, 37.8\% for Group 2, and $49.2 \%$ for Group 3. These results were statistically significant. Conclusions. The study demonstrates that amniotic membrane products have potential to provide bridging of bone defects. Filling bone defects without harvesting autogenous bone would provide a significant improvement in patient care.
\end{abstract}

\section{Introduction}

Although autologous iliac crest bone graft (AICBG) remains the "gold standard" in the reconstruction of bone defects, there are disadvantages, including a limited amount of bone and donor site morbidity [1]. Many bone graft substitutes have therefore been developed, including silicone, polymethylmethacrylate (PMMA) [2], porous polyethylene [3], hydroxyapatite, demineralized bone matrix (DBM), and tricalcium phosphate [4]. As foreign bodies, however, these alloplastic materials have their own inherent disadvantages, including increased infection and extrusion rates. Recent developments in the treatment of bone defects include the reamer irrigator aspirator system (RIA), Masquelet technique (induced membrane), bone marrow aspirate concentrate (BMAC), and BMP-2 combined with cancellous allograft [1]. Although novel, these new techniques lack high-level evidence for their widespread adoption. Tissue engineering has provided an attractive alternative to the use of synthetic implants and growth factors by using biodegradable materials of natural origin to form tissue constructs. The advantage to the tissue engineered approach to bone repair as reported in this study is the use of materials that are rapidly degrading and nonimmunogenic.

The objective of this study was to evaluate a novel biomaterial, already FDA approved for other musculoskeletal reconstructions for the repair of bone fractures and resections. Currently, autogenous bone grafts, allogenic bone grafts, or bone graft substitutes are used for such repairs, but these methods have the limitations and disadvantages described previously. The biomaterial evaluated is amniotic membrane-derived allograft. This material has been shown to be rich in growth factors that can direct mesenchymal stem cell differentiation. It is known to be quantitatively much higher in concentration of growth factors per unit volume compared to human embryonic stem cells as well as human bone marrow-derived mesenchymal stromal cells [5]. These factors make it highly attractive for clinical use. We have previously shown the addition of circulating stem cells to significantly improve the biomechanical strength of 
rat Achilles tendons following transection and repair [6]. A much broader application of this technology would be for repair of long bone fractures, spinal fusion, and resections.

In this study we implanted this biomaterial in rat femurs using the rat critical size femoral gap model and evaluated bone regeneration through radiographs of defect sites and histology studies.

\section{Materials and Methods}

2.1. Study Design. After creation of the critical size femoral gap defects animals were randomized to one of the following three groups: (1) control, which did not receive any treatment and was left empty; (2) bone graft alone, which was filled with a commercially available bone graft material; (3) bone graft plus the addition of $\mathrm{NuCel}$ amniotic tissue preparation.

2.2. Animal Model. Twenty-one adult male Sprague-Dawley rats $(300-400 \mathrm{~g})$ were acquired and acclimated in individual cages for at least one week before beginning surgical procedures. The use of animals was in accordance with protocols approved by the Institutional Animal Care and Use Committee at the North Shore-Long Island Jewish Health System/the Feinstein Institute for Medical Research.

2.3. Surgical Technique. The animals were anesthetized in accordance with IACUC protocol. The skin overlying the femur was shaved using surgical clippers. With the use of aseptic technique, a longitudinal skin incision was made directly over the femur. One hind limb was randomly assigned to either a control or experimental group. The femur was exposed by longitudinal incision and isolated. A HMW polyethylene fracture fixation plate was attached to the femur by 4 threaded Kirschner wires and two cerclage wires. After rigid fixation of the plate, an $8 \mathrm{~mm}$ transverse mid diaphyseal bone segment was removed by using a rotary osteotomy burr along with the adherent periosteum. The critical size gap is typically the same as the bone diameter. The defect was either left empty as a control or packed with either a commercial bone graft preparation (positive control) or a bone graft preparation mixed with amniotic-derived tissue (experimental group). Following treatment the muscles are opposed with the fascia. Skin closure was performed in a routine manner with use of 4-0 Vicryl sutures. The animals were not immobilized postoperatively. At 6 weeks after surgery, the animals were sacrificed and the femurs were harvested for radiographic assessment followed by formalin fixation and processing for histology.

2.4. Histology Image Analysis Methods. All samples were stained with Mallory's trichrome and were observed using Olympus model $\mathrm{BH}-2$ at $4 \mathrm{x}$ magnification (40x total magnification) in order to identify regions of interest (ROI) exhibiting new bone growth at the site of the original defect site. Upon identification, these areas were then observed under 100x total magnification. Multiple pictures were taken in order to encompass both the area of new bone growth and the surrounding extant bone matrix $(n \leq 5)$. All images were taken at $1360 \times 1024$ pixel resolution.

Each image was loaded into ImageJ (National Institute of Health, Bethesda, Maryland, USA) in order to determine the percentage of new bone growth present in the observed area. Once each region was selected, area measurements were made. The new bone growth area was calculated as a percentage of the total area. Mean percentage area and standard deviation for each sample group was calculated. A one-way analysis of variance (ANOVA) was performed between the 3 groups using IBM SPSS Statistics software $(\alpha=0.05)$.

\section{Results}

The surgery was well tolerated but there was one animal per group lost to follow-up due to failure of the hardware. The control, empty group exhibited little bone filling upon radiographic imaging. In contrast both experimental groups demonstrated excellent conduction of new bone formation across the critical size gap (Figure 1).

Histological analysis of the control samples showed a partially organized network of tissue containing a mixture of cells including marrow elements as well as inflammatory cells. There was little evidence of bone filling although some woven bone was detected at the defect margins (Figure 2). Group 2 bone graft samples showed improved osteoconduction with partial bridging of the osteotomy site. Typically there remained a small layer of cartilage within the middle of the callus with woven bone surrounding the fragments of bone graft (Figure 3). In contrast, group 3 showed near complete bridging of the defect gap with abundant periosteal woven bone formation observed (Figure 4). Quantitative histology demonstrated that Group 1 empty controls had an average of $11.1 \%$ new bone formation in the defect site. Group 2 showed an improved new bone formation rate of $37.8 \%$. Group 3 achieved the highest new bone formation rate of $49.2 \%$. The results of the one-way ANOVA showed that there was a significant difference between the 3 groups $(P<0.001)$. Post hoc Tukey's HSD test showed that there was a significant difference between Groups 3 and $1(P<0.001)$. However, there was only a marginal difference between Groups 2 and $3(P=0.062)$ (Figure 5).

\section{Discussion}

The clinical experience of treatment of osseous bone defects with autografts has had mixed results. While many studies have been shown to yield good to excellent results [7-9], several trials have reported mixed outcomes using autologous bone grafting. Rates of union in a trial of comminuted forearm fracture were equivocal in those treated with or without autograft $[10,11]$. Patients treated for tibial nonunions with autograft [12], while reporting a union rate of $85 \%$ still had significant deformity in the repair.

The primary limitation of autografts is the persistent morbidity associated with the donor harvest site [13]. The most common complication is pain at the donor site, which can be as high as $50 \%$ and lasting as long as one year in $29 \%$ 


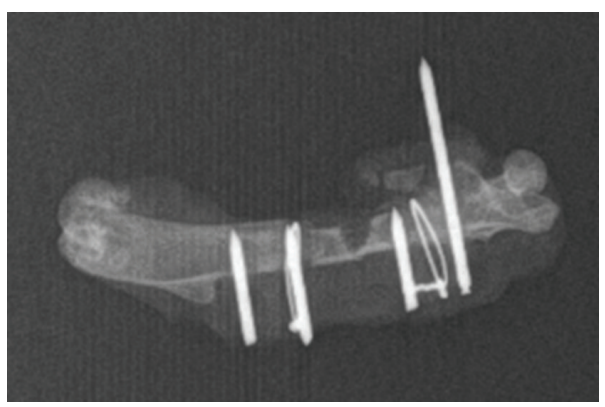

(a)

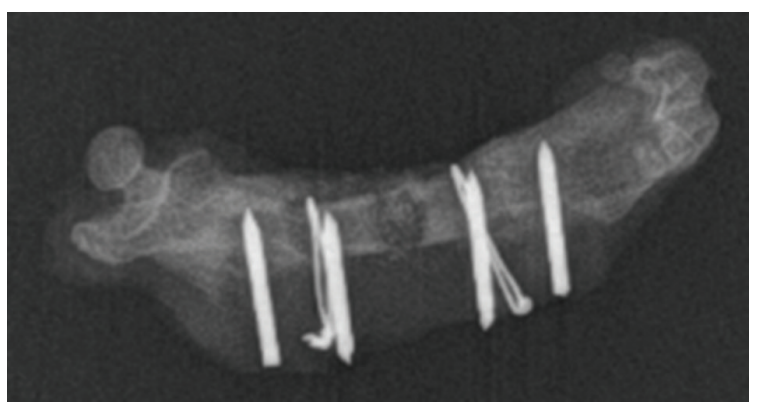

(b)

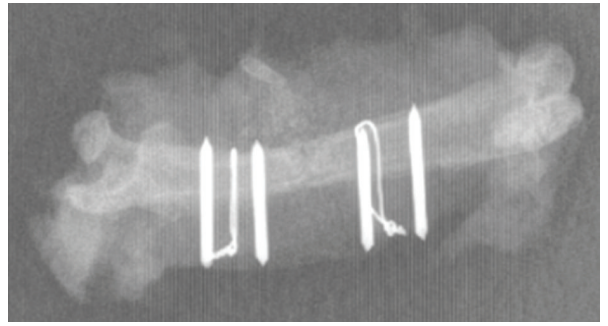

(c)

FIGURE 1: Radiographs of critical size femoral gap defects in the rat. (a) Control group 1 empty defect. Note the lack of any significant bone present. (b) Group 2 bone graft alone; new bone is present but not bridging. (c) Group 3 bone graft plus the addition of amniotic membrane allograft with robust bone formation and complete bridging of defect gap.

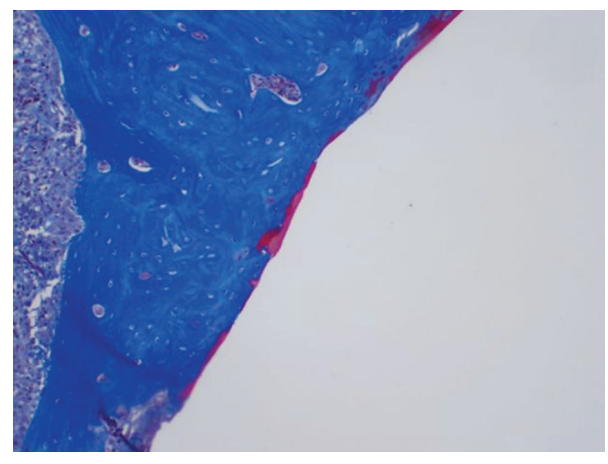

FIGURE 2: Photomicrograph of control Group 1 defect; prominent gap is present with no new bone formation. Mallory's trichrome $\times 200$ original magnification.

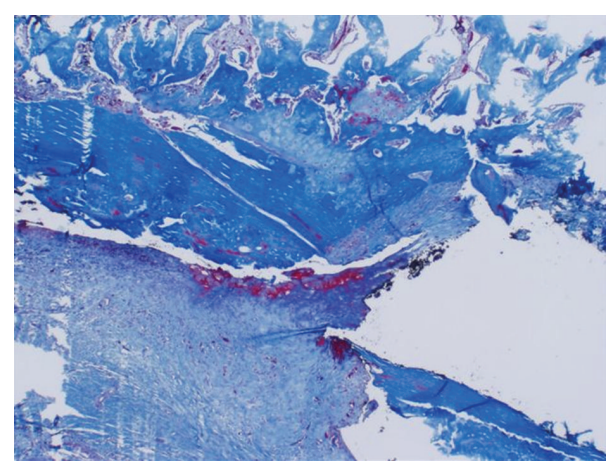

FIGURE 3: Photomicrograph of Group 2 bone graft alone; bone conduction is present adjacent to the bone graft granules. Mallory's trichrome $\times 200$ original magnification.

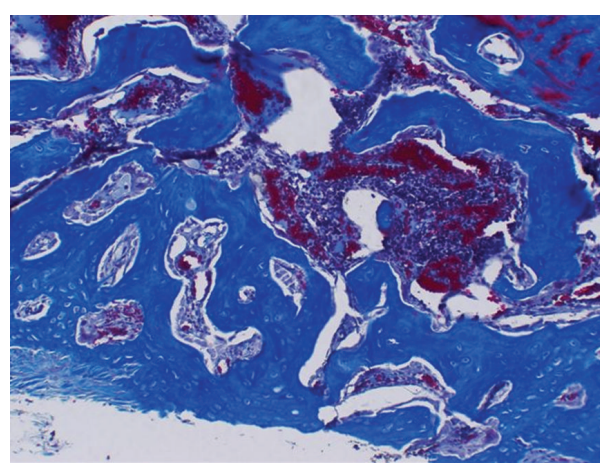

FIGURE 4: Photomicrograph of Group 3 bone graft plus amniotic membrane allograft; note robust new bone formation throughout the defect site. Mallory's trichrome $\times 200$ original magnification.

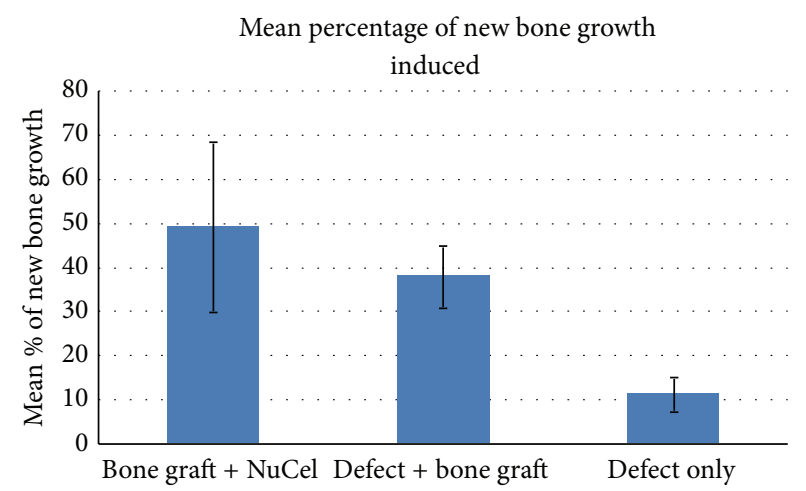

FIGURE 5: Graph of quantitative histological results using image analysis defining new bone formation among the groups tested. 
of patients $[7,14]$. These findings have increased the need for new materials for bone graft.

Our results demonstrated strong radiographic differences among the groups although it did not demonstrate a significant histological difference between bone graft and bone graft plus the addition of amniotic allograft tissue. We attribute this finding to several factors including the small $n$ enrolled and the surgical dropouts from the groups leading to a loss of statistical power. Other factors may be extravasation of the material away from the defect site leading to loss of a critical concentration of active material.

In this study we examined the use of a new technology utilizing amniotic-derived allograft material combined with bone graft. Amnion-based materials are a rich source of cytokines, growth factors, and hyaluronic acid after childbirth. Orthopaedic surgeons continually seek advanced techniques and technologies to increase positive surgical outcomes. Thus, using amniotic-based materials to aid in fracture healing of large boney defects is a potential alternative to autologous bone graft. Further research will be required to define the exact molecular mechanism of action; however, amniotic membrane growth factors are a promising possible alternative that may one day become part of the orthopaedic surgeons' armamentarium.

\section{Conflict of Interests}

No conflict of interests were declared by the authors.

\section{References}

[1] A. Nauth, M. D. McKee, T. A. Einhorn, J. T. Watson, R. Li, and E. H. Schemitsch, "Managing bone defects," Journal of Orthopaedic Trauma, vol. 25, no. 8, pp. 462-466, 2011.

[2] R. Murugan and S. Ramakrishna, "Development of nanocomposites for bone grafting," Composites Science and Technology, vol. 65, no. 15-16, pp. 2385-2406, 2005.

[3] J. J. Romano, N. T. Iliff, and P. N. Manson, "Use of medpor porous polyethylene implants in 140 patients with facial fractures," Journal of Craniofacial Surgery, vol. 4, no. 3, pp. 142-147, 1993.

[4] R. W. Bucholz, A. Carlton, and R. E. Holmes, "Hydroxyapatite and tricalcium phosphate bone graft substitutes," Orthopedic Clinics of North America, vol. 18, no. 2, pp. 323-334, 1987.

[5] A. J. Marcus, T. M. Coyne, J. Rauch, D. Woodbury, and I. B. Black, "Isolation, characterization, and differentiation of stem cells derived from the rat amniotic membrane," Differentiation, vol. 76, no. 2, pp. 130-144, 2008.

[6] R. J. Daher, N. O. Chahine, P. Razzano, S. A. Patwa, N. J. Sgaglione, and D. A. Grande, "Tendon repair augmented with a novel circulating stem cell population," International Journal of Clinical and Experimental Medicine, vol. 4, no. 3, pp. 214-219, 2011.

[7] G. P. Rajan, J. Fornaro, O. Trentz, and R. Zellweger, "Cancellous allograft versus autologous bone grafting for repair of comminuted distal radius fractures: a prospective, randomized trial," Journal of Trauma-Injury, Infection and Critical Care, vol. 60, no. 6, pp. 1322-1329, 2006.

[8] D. Ring, C. Allende, K. Jafarnia, B. T. Allende, and J. B. Jupiter, "Ununited diaphyseal forearm fractures with segmental defects: plate fixation and autogenous cancellous bone-grafting," Journal of Bone and Joint Surgery A, vol. 86, no. 11, pp. 2440-2445, 2004.

[9] P. E. Scranton Jr., C. C. Frey, and K. S. Feder, "Outcome of osteochondral autograft transplantation for type-V cystic osteochondral lesions of the talus," Journal of Bone and Joint Surgery B, vol. 88, no. 5, pp. 614-619, 2006.

[10] R. R. Wright, G. J. Schmeling, and J. P. Schwab, “The necessity of acute bone grafting in diaphyseal forearm fractures: a retrospective review," Journal of Orthopaedic Trauma, vol. 11, no. 4, pp. 288-294, 1997.

[11] S. Y. Wei, C. T. Born, A. Abene, A. Ong, R. Hayda, and W. G. DeLong Jr., "Diaphyseal forearm fractures treated with and without bone graft," Journal of Trauma-Injury, Infection and Critical Care, vol. 46, no. 6, pp. 1045-1048, 1999.

[12] D. H. Gershuni and R. Pinsker, "Bone grafting for nonunion of fractures of the tibia: a critical review," Journal of Trauma, vol. 22, no. 1, pp. 43-49, 1982.

[13] J. L. Russell and J. E. Block, "Surgical harvesting of bone graft from the ilium: point of view," Medical Hypotheses, vol. 55, no. 6, pp. 474-479, 2000.

[14] J. C. Fernyhough, J. J. Schimandle, M. C. Weigel, C. C. Edwards, and A. M. Levine, "Chronic donor site pain complicating bone graft harvesting from the posterior iliac crest for spinal fusion," Spine, vol. 17, no. 12, pp. 1474-1480, 1992. 


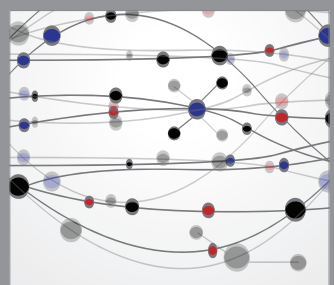

The Scientific World Journal
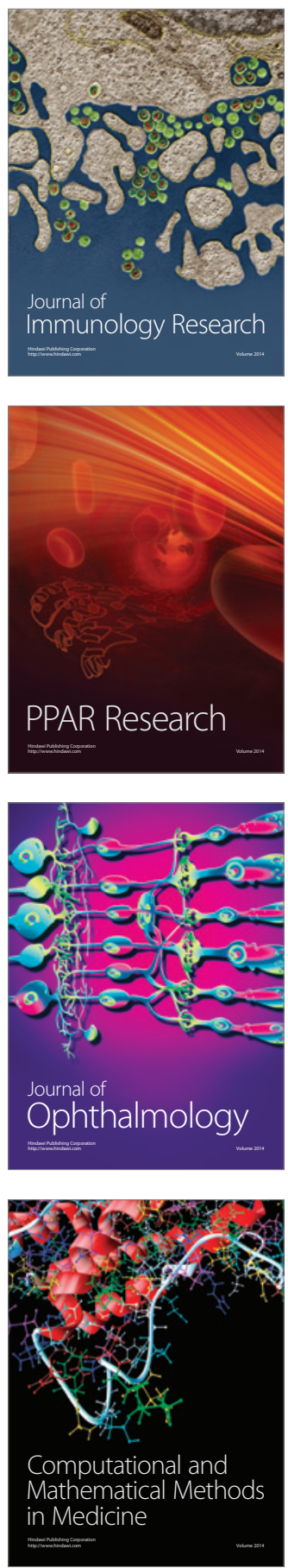

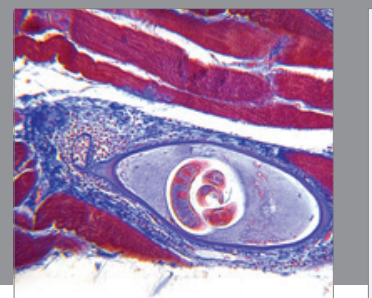

Gastroenterology

Research and Practice
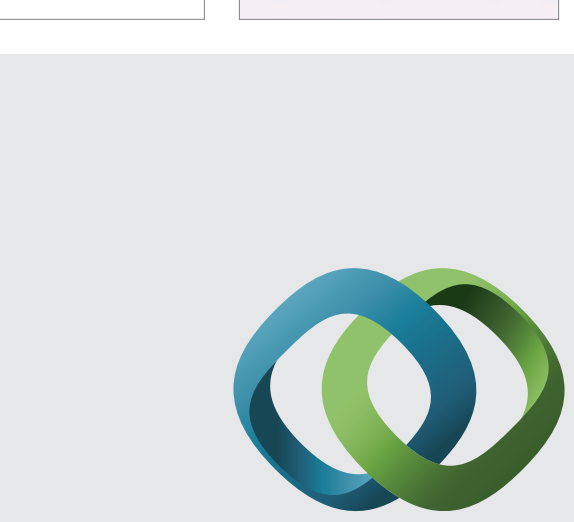

\section{Hindawi}

Submit your manuscripts at

http://www.hindawi.com
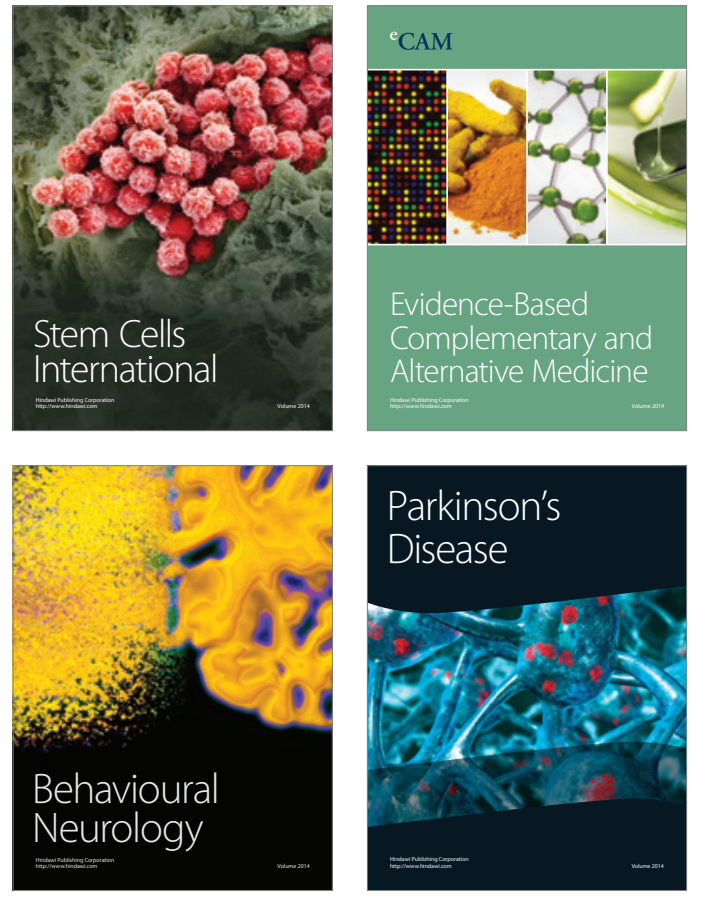
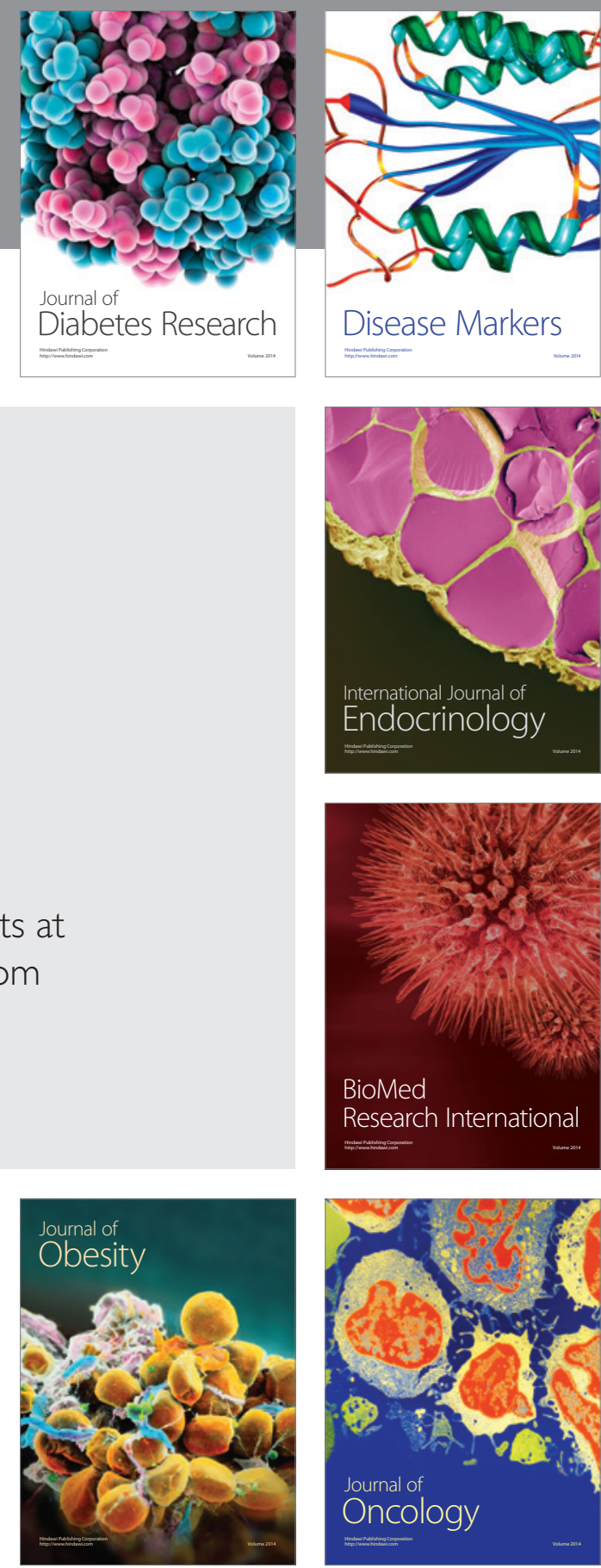

Disease Markers
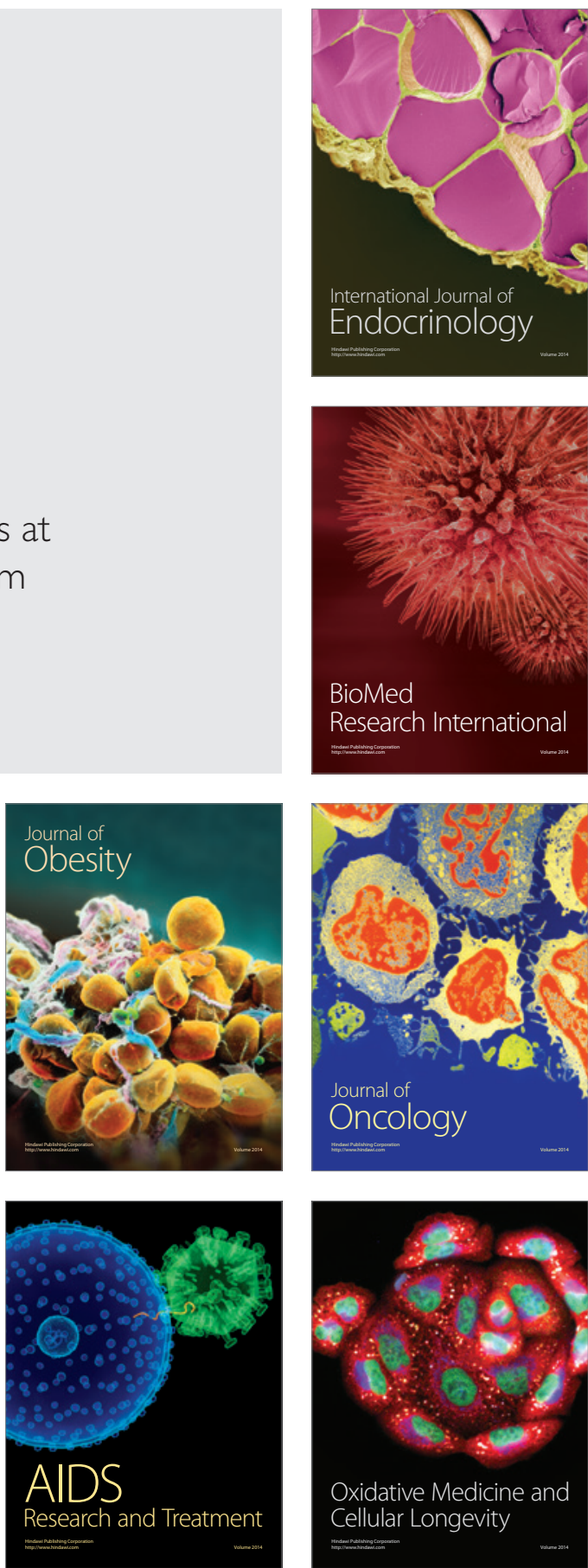